\title{
Exponential behavior of solutions to stochastic integrodifferential equations with distributed delays
}

\author{
Mamadou Abdoul DIOP ${ }^{a 1}$, Tomás CARABALLO ${ }^{b}$ and Abdoul Aziz \\ NDIAYE ${ }^{c}$
}

\author{
${ }^{a}$ Université Gaston Berger \\ UFR des Sciences Appliquées et Technologie, Département de Mathématiques \\ BP 234, Saint-louis, Sénégal \\ e-mail: mamadou-abdoul.diop@ugb.edu.sn \\ ${ }^{b}$ Dpto. Ecuaciones Diferenciales y Análisis Numérico \\ Universidad de Sevilla, Apdo. de Correos 1160, 41080-Sevilla, Spain \\ e-mail: caraball@us.es \\ ${ }^{c}$ Université Gaston Berger \\ UFR des Sciences Appliquées et Technologie, Département de Mathématiques \\ BP 234, Saint-louis, Sénégal \\ e-mail: azizndiaye@yahoo.com
}

\begin{abstract}
In this work, we study the existence, uniqueness and exponential asymptotic behavior of mild solutions to stochastic integrodifferential delay evolution equations. We assume that the non-delay part generates a $C_{0}$-semigroup.
\end{abstract}

Keywords: $\quad C_{0^{-}}$semigroup, partial functional differential equations, Brownian motion, finite delay, mild solution, exponential decay in mean square.

\section{Introduction}

In the last two decades, stochastic differential equations have attracted many physicists, mathematicians and engineers, and remarkable contributions have been made to both theory and applications of stochastic partial differential equations with delays. The stochastic functional differential systems appears in many areas of sciences. Such equations arise in the study of stochastic systems in the presence of hereditary influences on the state variable. For example, stochastic integrodifferential systems which cover a large area of system dynamics including reactor dynamics $[4,16,18]$, heat transfer by conduction and radiation $[15,19]$, mathematical modeling of system hysteresis $[8,16]$, models of transmission of infections or diseases [3], etc. The presence of a stochastic component makes the evolution of the state variable a stochastic process and accounts for intrinsic or external random influences. One of the fundamental problems in such dynamical systems is to establish sufficient conditions under which it exhibits a unique solution. There are many ways to establish such results, for example: (i) Monotone Iterative Techniques, which is

\footnotetext{
${ }^{1}$ To whom all correspondence should be sent: mamadou-abdoul.diop@ugb.edu.sn
} 
based in the method of upper and lower solutions [15], used mainly for boundary value problems, (ii) Topological Methods, which use one of the fixed point theorems such as Schauder-Tychonoff's [6], and (iii) Construction Methods, such as the Picard successive approximations $[17,2,1,11,13,14,20]$ in which one has to show that the convergence of the approximation process to the original solution.

Recently, Caraballo et al. [5] have studied the following class of stochastic delay evolution equations with fractional Brownian motion

$$
\left\{\begin{array}{l}
d x(t)=\left[A x(t)+f\left(t, x_{t}\right)\right] d t+g(t) d B_{Q}^{H}(t) \text { for } t>0 \\
x(t)=\varphi(t), \quad t \in[-r, 0], \quad(0 \leq r<\infty)
\end{array}\right.
$$

with Hurst parameter $H \in\left(\frac{1}{2}, 1\right)$. They investigated the existence, uniqueness and exponential behavior of mild solutions to (1.1).

In this paper we consider the following stochastic integrodifferential equation,

$$
\left\{\begin{array}{l}
d x(t)=A x(t) d t+\left[\int_{0}^{t} B(t-s) x(s) d s+F\left(t, x_{t}\right)\right] d t+G\left(t, x_{t}\right) d w(t), \text { for } t \in[0, T] \\
x(s)=\varphi, \quad-r \leq s \leq 0, \quad r \geq 0
\end{array}\right.
$$

under suitable assumptions on the operator $A$, the coefficients $F, G$, and the initial value $\varphi$. Here $w(t)$ denotes a Brownian motion.

The schemes of the proofs are similar to those ones in [5] but with necessary modifications due to the different nature of the problem. To be more precise, we suppose that the non-delay part admits a resolvent operator in the sense given by Grimmer[9], as well as the noise considered in [5] is a fractional Brownian motion while the one in this paper is a Hilbert-valued Wiener process. The main objective of this paper is to investigate existence and uniqueness of mild solutions to the stochastic integrodifferential equation (1.2), and to study its long-time behaviour as well, by using the theory of resolvent operators and Picard type iteration. Recall that the resolvent operator plays an important role in solving Eq. (1.2) in the weak and strict sense, it replaces the role of the $C_{0}$-semigroup theory. For more details we refer to [10, 9].

The paper is organized as follows: in Section 2 we recall some preliminaries which are used throughout this paper. In Section 3 we state the existence and uniqueness of a mild solution. In Section 4 we study the asymptotic behaviour of (1.2). Finally, Section 5 is devoted to show an example to illustrate the efficiency of the obtained results.

\section{Preliminaries}

\subsection{Wiener process}

Throughout this paper, let $\mathbb{H}$ and $\mathbb{K}$ be two real separable Hilbert spaces. We denote by $\langle\cdot, \cdot\rangle_{\mathbb{H}},\langle\cdot, \cdot\rangle_{\mathbb{K}}$ their inner products and by $\|\cdot\|_{\mathbb{H}},\|\cdot\|_{\mathbb{K}}$ their vectors norms, respectively. $\mathcal{L}(\mathbb{H}, \mathbb{K})$ denote the space of all bounded linear operators from $\mathbb{H}$ into $\mathbb{K}$, equipped with the usual operator norm $\|\cdot\|$. If $\mathbb{H}=\mathbb{K}$ we will simply write $\mathcal{L}(\mathbb{H})$ instead of 
$\mathcal{L}(\mathbb{H}, \mathbb{H})$. In the sequel, we always use the same symbol $\|\cdot\|$ to denote norms of operators regardless of the spaces potentially involved when no confusion possibly arises. Moreover, let $\left(\Omega, \mathcal{F},\left\{\mathcal{F}_{t}\right\}_{t \geq 0}, \mathbb{P}\right)$ be a complete probability space with a normal filtration $\left\{\mathcal{F}_{t}\right\}_{t \geq 0}$ satisfying the usual conditions (i.e. it is increasing and right-continuous, while $\mathcal{F}_{0}$ contains all $\mathbb{P}$-null sets). Let $\{w(t): t \geq 0\}$ denote a $\mathbb{K}$-valued Wiener process defined on the probability space $\left(\Omega, \mathcal{F},\left\{\mathcal{F}_{t}\right\}_{t \geq 0}, \mathbb{P}\right)$, with covariance operator Q, that is, $E\langle w(t), x\rangle_{\mathbb{K}}\langle w(s), y\rangle_{\mathbb{K}}=(t \wedge s)\langle Q x, y\rangle_{\mathbb{K}}$, for all $x, y \in \mathbb{K}$, where $\mathrm{Q}$ is a positive, selfadjoint, trace class operator on $\mathbb{K}$. In particular, we denote by $w(t)$ a $\mathbb{K}$-valued $Q$-Wiener process with respect to $\left\{\mathcal{F}_{t}\right\}_{t \geq 0}$. To define stochastic integrals with respect to the $Q_{-}$ Wiener process $w(t)$, we introduce the subspace $\mathbb{K}_{0}=Q^{1 / 2} \mathbb{K}$ of $\mathbb{K}$ endowed with the inner product $\langle u, v\rangle_{\mathbb{K}_{0}}=\left\langle Q^{-1 / 2} u, Q^{-1 / 2} v\right\rangle_{\mathbb{K}}$ as a Hilbert space. We assume that there exists a complete orthonormal system $\left\{e_{i}\right\}$ in $\mathbb{K}$, a bounded sequence of positive real numbers $\lambda_{i}$ such that $Q e_{i}=\lambda_{i} e_{i}, \quad i=1,2, \ldots$, and a sequence $\left\{w_{i}(t)\right\}_{i>1}$ of independent standard Brownian motions such that

$w(t)=\sum_{i=1}^{+\infty} \sqrt{\lambda_{i}} w_{i}(t) e_{i}$ for $t \geq 0$ and $\mathcal{F}_{t}=\mathcal{F}_{t}^{w}$, where $\mathcal{F}_{t}^{w}$ is the $\sigma$-algebra generated by $\{w(s): 0 \leq s \leq t\}$. Let $\mathcal{L}_{2}^{0}=\mathcal{L}_{2}\left(\mathbb{K}_{0}, \mathbb{H}\right)$ be the space of all Hilbert-Schmidt operators from $\mathbb{K}_{0}$ to $\mathbb{H}$. It turns out to be a separable Hilbert space equipped with the norm $\|v\|_{\mathcal{L}_{2}^{0}}=\operatorname{tr}\left(\left(v Q^{1 / 2}\right)\left(v Q^{1 / 2}\right)^{*}\right)$ for any $v \in \mathcal{L}_{2}^{0}$. Obviously, for any bounded operator $v \in \mathcal{L}_{2}^{0}$, this norm reduces to $\|v\|_{\mathcal{L}_{2}^{0}}^{2}=\operatorname{tr}\left(v Q v^{*}\right)$.

\subsection{Partial integro-differential equations in Banach spaces}

In the present section, we recall some definitions, notations and properties needed in the sequel.

In what follows, $\mathbb{H}$ will denote a Banach space, $A$ and $B(t)$ are closed linear operators on $\mathbb{H}$. $Y$ represents the Banach space $D(A)$, the domain of operator $A$, equipped with the graph norm

$$
|y|_{Y}:=|A y|+|y| \quad \text { for } y \in Y .
$$

The notation $C([0,+\infty) ; Y)$ stands for the space of all continuous functions from $[0,+\infty)$ into $Y$. We then consider the following Cauchy problem

$$
\left\{\begin{array}{l}
v^{\prime}(t)=A v(t)+\int_{0}^{t} B(t-s) v(s) d s \text { for } t \geq 0 \\
v(0)=v_{0} \in \mathbb{H} .
\end{array}\right.
$$

Definition 2.1. ([9]) A resolvent operator for Eq. (2.1) is a bounded linear operator valued function $R(t) \in \mathcal{L}(\mathbb{H})$ for $t \geq 0$, satisfying the following properties :

(i) $R(0)=I$ and $|R(t)| \leq N e^{\beta t}$ for some constants $N$ and $\beta$.

(ii) For each $x \in \mathbb{H}, R(t) x$ is strongly continuous for $t \geq 0$.

(iii) For $x \in Y, R(\cdot) x \in C^{1}([0,+\infty) ; \mathbb{H}) \cap C([0,+\infty) ; Y)$ and 


$$
\begin{aligned}
R^{\prime}(t) x & =A R(t) x+\int_{0}^{t} B(t-s) R(s) x d s \\
& =R(t) A x+\int_{0}^{t} R(t-s) B(s) x d s \quad \text { for } t \geq 0 .
\end{aligned}
$$

For additional details on resolvent operators, we refer the reader to [21, 9]. The resolvent operator plays an important role to study the existence of solutions and to establish a variation of constants formula for non-linear systems. For this reason, we need to know when the linear system (2.1) possesses a resolvent operator. Theorem 2.2 below provides a satisfactory answer to this problem.

In what follows we suppose the following assumptions:

(H1) $\mathrm{A}$ is the infinitesimal generator of a $C_{0}$-semigroup $(T(t))_{t \geq 0}$ on $\mathbb{H}$.

(H2) For all $t \geq 0, B(t)$ is a continuous linear operator from $\left(Y,|\cdot|_{Y}\right)$ into $\left(\mathbb{H},|\cdot|_{\mathbb{H}}\right)$. Moreover, there exists an integrable function $c:[0,+\infty) \rightarrow \mathbb{R}^{+}$such that for any $y \in Y$, $y \mapsto B(t) y$ belongs to $W^{1,1}([0,+\infty), \mathbb{H})$ and

$$
\left|\frac{d}{d t} B(t) y\right|_{\mathbb{H}} \leq c(t)|y|_{Y} \text { for } y \in Y \text { and } t \geq 0 .
$$

Theorem 2.2. ([7]) Assume that hypotheses (H1) and (H2) hold. Then Eq. (2.1) admits a resolvent operator $(R(t))_{t \geq 0}$.

Theorem 2.3. ([12]) Assume that hypotheses (H1) and (H2) hold. Let $T(t)$ be a compact operator for $t>0$. Then, the corresponding resolvent operator $R(t)$ of Eq. is continuous for $t>0$ in the operator norm, namely, for all $t_{0}>0$, it holds that $\lim _{h \rightarrow 0}\left\|R\left(t_{0}+h\right)-R\left(t_{0}\right)\right\|=0$.

In the sequel, we recall some results on the existence of solutions for the following integro-differential equation

$$
\left\{\begin{array}{l}
v^{\prime}(t)=A v(t)+\int_{0}^{t} B(t-s) v(s) d s+q(t) \text { for } t \geq 0 \\
v(0)=v_{0} \in \mathbb{H}
\end{array}\right.
$$

where $q:[0,+\infty[\rightarrow \mathbb{H}$ is a continuous function.

Definition 2.4. ([9]) A continuous function $v:[0,+\infty) \rightarrow \mathbb{H}$ is said to be a strict solution of Eq. (2.2) if

(i) $v \in C^{1}([0,+\infty) ; \mathbb{H}) \cap C([0,+\infty) ; Y)$,

(ii) $v$ satisfies Eq. (2.2) for $t \geq 0$.

Remark 2.5. From this definition we deduce that $v(t) \in D(A)$, and the function $B(t-s) v(s)$ is integrable, for all $t>0$ and $s \in[0,+\infty)$. 
Theorem 2.6. ([9]) Assume that (H1)-(H2) hold. If $v$ is a strict solution of Eq. (2.2), then the following variation of constants formula holds

$$
v(t)=R(t) v_{0}+\int_{0}^{t} R(t-s) q(s) d s \quad \text { for } \quad t \geq 0 .
$$

Accordingly, we can establish the following definition.

Definition 2.7. ([9]) A function $v:[0,+\infty) \rightarrow \mathbb{H}$ is called a mild solution of $(2.2)$, for $v_{0} \in \mathbb{H}$, if $v$ satisfies the variation of constants formula (2.3).

The next theorem provides sufficient conditions ensuring the regularity of solutions of Eq. (2.2).

Theorem 2.8. ([9]) Let $q \in C^{1}([0,+\infty) ; \mathbb{H})$ and $v$ be defined by $(2.3)$. If $v_{0} \in D(A)$, then $v$ is a strict solution of Eq. (2.2).

\section{Existence and uniqueness of mild solution}

Consider $(\Omega, \mathcal{F}, \mathbb{P})$ the complete probability space which was introduce in Section 2 . Denote $\mathcal{F}_{t}=\mathcal{F}_{0}$, for all $t \leq 0$. We denote by $C\left(a, b ; L^{2}(\Omega ; \mathbb{H})\right)=C\left(a, b ; L^{2}(\Omega, \mathcal{F}, \mathbb{P} ; \mathbb{H})\right)$ the Banach space of all continuous functions from $[a, b]$ into $L^{2}(\Omega ; \mathbb{H})$ equipped with the sup norm.

Let us also consider two fixed real numbers $r \geq 0$ and $T>0$. If $x \in C\left(-r, T ; L^{2}(\Omega ; \mathbb{H})\right)$, for each $t \in[0, T]$ we denote by $x_{t} \in C\left(-r, 0 ; L^{2}(\Omega ; \mathbb{H})\right)$ the function defined by $x_{t}(s)=x(t+s)$ for $s \in[-r, 0]$.

In this section we consider the existence and uniqueness of mild solutions to the following stochastic integrodifferential equation with delays:

$$
\left\{\begin{array}{l}
d X(t)=\left(A X(t)+\int_{0}^{t} B(t-s) X(s) d s+f\left(t, X_{t}\right)\right) d t+g\left(t, X_{t}\right) d w(t) \quad t \in[0, T], \\
X(t)=\varphi(t) \text { for } t \in[-r, 0]
\end{array}\right.
$$

where $w(t)$ is the Brownian motion which was introduced in the previous section, the initial data $\varphi \in C\left(-r, 0 ; L^{2}(\Omega ; \mathbb{H})\right)$ and $A: \operatorname{Dom}(A) \subset \mathbb{H} \rightarrow \mathbb{H}$ is the infinitesimal generator of a strongly continuous semigroup $S(\cdot)$ on $\mathbb{H}$, that is, for $t \geq 0$, it holds,

$$
|S(t)| \leq M e^{\rho t}, \quad M \geq 1, \rho \in \mathbb{R} .
$$

Let $f:[0, T] \times C(-r, 0 ; \mathbb{H}) \rightarrow \mathbb{H}$ and $g:[0, T] \times C(-r, 0 ; \mathbb{H}) \rightarrow \mathcal{L}_{2}^{0}(\mathbb{K}, \mathbb{H})$ be families of nonlinear operators, defined for almost every $t$ (a.e. $t$ ), satisfying

(H3) The mapping $t \in(0, T) \rightarrow f(t, \xi) \in \mathbb{H}$ is Lebesgue measurable, for a.e. $t$, and for all $\xi \in C(-r, 0 ; \mathbb{H})$. 
(H4) There exists a constant $C_{f}$, which is independent of $T$, such that for any $x, y \in C(-r, T ; \mathbb{H})$ and $t \in[0, T]$,

$$
\int_{0}^{t}\left\|f\left(s, x_{s}\right)-f\left(s, y_{s}\right)\right\|_{\mathbb{H}}^{2} d s \leq C_{f} \int_{-r}^{t}\|x(s)-y(s)\|_{\mathbb{H}}^{2} d s
$$

(H5)

$$
\int_{0}^{T}\|f(s, 0)\|_{\mathbb{H}}^{2} d s<\infty .
$$

(H6) The mapping $t \in(0, T) \rightarrow g(t, \xi) \in \mathbb{H}$ is Lebesgue measurable, for a.e. $t$, and for all $\xi \in C(-r, 0 ; \mathbb{H})$.

(H7) There exists a constant $C_{g}$ such that for any $x, y \in C(-r, T ; \mathbb{H})$ and $t \in[0, T]$,

$$
\int_{0}^{t}\left\|g\left(s, x_{s}\right)-g\left(s, y_{s}\right)\right\|_{\mathcal{L}_{2}^{0}}^{2} d s \leq C_{g} \int_{-r}^{t}\|x(s)-y(s)\|_{\mathbb{H}}^{2} d s
$$

(H8)

$$
\int_{0}^{T}\|g(s, 0)\|_{\mathcal{L}_{2}^{0}}^{2} d s<\infty
$$

Definition 3.1. Let $(R(t))_{t \geq 0}$ be a resolvent operator for Eq. (2.1). A $\mathbb{H}$-valued process $X(t)$ is called a mild solution of (3.1) if $X \in C\left(-r, T ; L^{2}(\Omega ; \mathbb{H})\right), X(t)=\varphi(t)$ for $t \in[-r, 0]$, and for $t \in[0, T]$, satisfies

$$
X(t)=R(t) \varphi(0)+\int_{0}^{t} R(t-s) f\left(s, X_{s}\right) d s+\int_{0}^{t} R(t-s) g\left(s, X_{s}\right) d w(s) \quad \mathbb{P}-\text { a.s. }
$$

Theorem 3.2. Suppose that assumptions (H1)-(H7) hold. Then, for every $\varphi \in C\left(-r, 0 ; L^{2}(\Omega ; \mathbb{H})\right)$, there exists a unique mild solution to (3.1).

Proof. We can assume that $\rho>0$, otherwise we can take $\rho_{0}>0$ such that $\|R(t)\| \leq M e^{\rho_{0} t}$, for all $t \geq 0$. We start by proving the uniqueness of mild solutions. Assume that $X, Y \in C\left(-r, T ; L^{2}(\Omega ; \mathbb{H})\right)$ are two mild solutions of (3.1). Then,

$$
\begin{aligned}
& \mathbb{E}\|X(t)-Y(t)\|_{\mathbb{H}}^{2} \leq 2 t \mathbb{E} \int_{0}^{t}\left\|R(t-s)\left(f\left(s, X_{s}\right)-f\left(s, Y_{s}\right)\right)\right\|_{\mathbb{H}}^{2} d s \\
&+2 t \mathbb{E} \int_{0}^{t}\left\|R(t-s)\left(g\left(s, X_{s}\right)-g\left(s, Y_{s}\right)\right)\right\|_{\mathcal{L}_{2}^{0}}^{2} d s \\
& \leq 2 t M^{2} e^{2 \rho t} \mathbb{E} \int_{0}^{t}\left\|f\left(s, X_{s}\right)-f\left(s, Y_{s}\right)\right\|_{\mathbb{H}}^{2} d s \\
& \quad+2 t M^{2} e^{2 \rho t} \mathbb{E} \int_{0}^{t}\left\|g\left(s, X_{s}\right)-g\left(s, Y_{s}\right)\right\|_{\mathcal{L}_{2}^{0}}^{2} d s \\
& \leq \quad\left[2 t M^{2} e^{2 \rho t} C_{f}+2 t M^{2} e^{2 \rho t} C_{g}\right] \int_{0}^{t} \mathbb{E}\|X(s)-Y(s)\|_{\mathbb{H}}^{2} d s \\
& \leq {\left[2 t M^{2} e^{2 \rho t} C_{f}+2 t M^{2} e^{2 \rho t} C_{g}\right] \int_{0}^{t} \sup _{0 \leq \tau \leq s} \mathbb{E}\|X(\tau)-Y(\tau)\|_{\mathbb{H}}^{2} d s, }
\end{aligned}
$$


and therefore, since $X=Y$ for $t \in[-r, 0]$, by taking the supremum in the above inequality,

$$
\sup _{0 \leq \theta \leq t} \mathbb{E}\|X(\theta)-Y(\theta)\|_{\mathbb{H}}^{2} \leq\left[2 T M^{2} e^{2 \rho t} C_{f}+2 T M^{2} e^{2 \rho t} C_{g}\right] \int_{0}^{t} \sup _{0 \leq \tau \leq s} \mathbb{E}\|X(\tau)-Y(\tau)\|_{\mathbb{H}}^{2} d s .
$$

The Gronwall Lemma ensures the uniqueness of solutions.

Now we prove the existence of solution to problem (3.1). First of all we check that the well-defined stochastic integral possesses the required regularity. To do that, let us consider $h>0$ small enough. We have

$$
\begin{aligned}
& E\left\|\int_{0}^{t+h} R(t+h-s) g\left(s, x_{s}\right) d w(s)-\int_{0}^{t} R(t-s) g\left(s, x_{s}\right) d w(s)\right\|^{2} \\
& \leq 2 E\left\|\int_{0}^{t}(R(t+h-s)-R(t-s)) g\left(s, x_{s}\right) d w(s)\right\|^{2}+2 E\left\|\int_{t}^{t+h} R(t-s) g\left(s, x_{s}\right) d w(s)\right\|^{2} \\
& :=I_{1}+I_{2}
\end{aligned}
$$

Firstly,

$$
I_{1} \leq 2\|R(t+h-s)-R(t-s)\| \int_{0}^{t}\left\|g\left(s, x_{s}\right)\right\|_{\mathcal{L}_{2}^{0}}^{2} d s \rightarrow 0 \text { when } h \rightarrow 0,
$$

thanks to the norm continuty of $R(t), t>0$.

Secondly,

$$
I_{2} \leq 2 M^{2} e^{\rho h}\left\|\int_{t}^{t+h} g\left(s, x_{s}\right)\right\|_{\mathcal{L}_{2}^{0}}^{2} d s \rightarrow 0 \text { when } h \rightarrow 0 .
$$

Therefore, the stochastic integral belongs to the space $C\left(-r, T ; L^{2}(\Omega ; \mathbb{H})\right)$.

We denote $X^{0}=0$ and define by recurrence a sequence $\left\{X^{n}\right\}_{n \in \mathbb{N}}$ of processes as

$$
\left\{\begin{array}{l}
X^{n}(t)=R(t) \varphi(0)+\int_{0}^{t} R(t-s) f\left(s, X_{s}^{n-1}\right) d s+\int_{0}^{t} R(t-s) g\left(s, X_{s}^{n-1}\right) d w(s), \quad t \in[0, T] \\
X^{n}(t)=\varphi(t), t \in[-r, 0] .
\end{array}\right.
$$

The sequence (3.3) is well defined, since $X^{0}=0 \in C\left(-r, T ; L^{2}(\Omega ; \mathbb{H})\right)$ and given $X^{n-1} \in C\left(-r, T ; L^{2}(\Omega ; \mathbb{H})\right)$, it is not difficult to prove that $X^{n} \in C\left(-r, T ; L^{2}(\Omega ; \mathbb{H})\right)$ as well.

Indeed, in order to prove the previous assertion, let us consider again $h>0$ sufficently 
small. Then,

$$
\begin{aligned}
\mathbb{E}\left\|X^{n}(t+h)-X^{n}(t)\right\|_{\mathbb{H}}^{2} \leq \quad & 5 \mathbb{E}\|(R(t+h-s)-R(t-s)) \varphi(0)\|^{2} \\
& +5 \mathbb{E}\left\|\int_{0}^{t}(R(t+h-s)-R(t-s)) f\left(s, X_{s}^{n}\right) d s\right\|_{\mathbb{H}}^{2} \\
& +5 \mathbb{E}\left\|\int_{t}^{t+h} R(t+h-s) f\left(s, X_{s}^{n}\right) d s\right\|_{\mathbb{H}}^{2} \\
& +5 \mathbb{E}\left\|\int_{0}^{t}(R(t+h-s)-R(t-s)) g\left(s, X_{s}^{n}\right) d w(s)\right\|_{\mathbb{H}}^{2} \\
& +5 \mathbb{E}\left\|\int_{t}^{t+h} R(t+h-s) g\left(s, X_{s}^{n}\right) d w(s)\right\|_{\mathbb{H}}^{2} \\
:= & I_{1}+I_{2}+I_{3}+I_{4}+I_{5} .
\end{aligned}
$$

We can easily show easily that $\mathbb{E} I_{1} \rightarrow 0$ when $h \rightarrow 0$.

Now, on the one hand,

$$
\mathbb{E} I_{2} \leq 5 \mathbb{E} \int_{0}^{t}\|R(t+h-s)-R(t-s)\|\left\|f\left(s, X_{s}^{n}\right) d s\right\|_{\mathbb{H}}^{2} \rightarrow 0 \text { when } h \rightarrow 0
$$

thanks to the norm continuty of $R(t)$ and the fact that

$$
\mathbb{E} \int_{0}^{t}\left\|f\left(s, X_{s}^{n}\right)\right\|_{\mathbb{H}}^{2} d s \leq C_{f} \mathbb{E} \int_{-r}^{t}\left\|X^{n-1}(s)\right\|_{\mathbb{H}}+\mathbb{E} \int_{0}^{t}\|f(s, 0)\|_{\mathbb{H}} d s<\infty
$$

due to Hypotheses $(\mathbf{H} 4)$ and $(\mathbf{H 5})$ and the fact that $X^{n-1} \in C\left(-r, T ; L^{2}(\Omega ; \mathbb{H})\right)$.

On the other hand,

$$
\begin{aligned}
I_{3} & \leq 5 h M^{2} e^{2 \rho h} \int_{t}^{t+h}\left\|f\left(s, X_{s}^{n-1}\right)-f(s, 0)\right\|_{\mathbb{H}}^{2} d s+5 h M^{2} e^{2 \rho h} \int_{t}^{t+h}\|f(s, 0)\|_{\mathbb{H}}^{2} d s \\
& \leq 5 h M^{2} e^{2 \rho h} C_{f} \int_{-r}^{t+h}\left\|X^{n-1}(s)\right\|_{\mathbb{H}}^{2} d s+5 h M^{2} e^{2 \rho h} \int_{t}^{t+h}\|f(s, 0)\|_{\mathbb{H}}^{2} d s,
\end{aligned}
$$

so that

$$
\mathbb{E} I_{3} \leq 5 h M^{2} e^{2 \rho h} C_{f} \int_{-r}^{t+h} \mathbb{E}\left\|X^{n-1}(s)\right\|_{\mathbb{H}}^{2} d s+5 h M^{2} e^{2 \rho h} \int_{t}^{t+h}\|f(s, 0)\|_{\mathbb{H}}^{2} d s \rightarrow 0
$$

when $h \rightarrow 0$.

As for the term $I_{4}$ we have,

$$
\mathbb{E} I_{4} \leq 5 \mathbb{E} \int_{0}^{t}\|R(t+h-s)-R(t-s)\|\left\|g\left(s, X_{s}^{n}\right)\right\|_{\mathcal{L}_{2}^{0}}^{2} d s \rightarrow 0, \text { when } h \rightarrow 0,
$$

thanks to the norm continuity of $R(t)$ and

$$
\mathbb{E} \int_{0}^{t}\left\|g\left(s, X_{s}^{n}\right)\right\|_{\mathcal{L}_{2}^{0}}^{2} d s \leq C_{g} \mathbb{E} \int_{-r}^{t}\left\|X^{n-1}(s)\right\|_{\mathbb{H}}+\mathbb{E} \int_{0}^{t}\|g(s, 0)\|_{\mathcal{L}_{2}^{0}} d s<\infty,
$$


due to Hypotheses (H6) and (H7) and the fact that $X^{n-1} \in C\left(-r, T ; L^{2}(\Omega ; \mathbb{H})\right)$. For the last term we have,

$$
\mathbb{E} I_{5} \leq 5 h M^{2} e^{2 \rho h} C_{g} \int_{-r}^{t+h} \mathbb{E}\left\|X^{n-1}(s)\right\|_{\mathbb{H}}^{2} d s+5 h M^{2} e^{2 \rho h} \int_{t}^{t+h}\|g(s, 0)\|_{\mathcal{L}_{2}^{0}}^{2} d s \rightarrow 0
$$

when $h \rightarrow 0$.

We want to show now that $\left\{X^{n}\right\}_{n \in \mathbb{N}}$ is a Cauchy sequence in $C\left(-r, T ; L^{2}(\Omega ; \mathbb{H})\right)$. Firstly, for $t \in[0, T]$ and $n \in \mathbb{N}$, since $X^{n}=Y^{n}$ on $[-r, 0]$, it holds

$$
\begin{aligned}
\left\|X^{n+1}(t)-X^{n}(t)\right\|_{\mathbb{H}}^{2} \leq & {\left[2 M^{2} t e^{2 \rho t} C_{f}\right] \int_{0}^{t}\left\|X^{n}(s)-X^{n-1}(s)\right\|_{\mathbb{H}}^{2} d s } \\
& +\left[2 M^{2} t e^{2 \rho t} C_{g}\right] \int_{0}^{t}\left\|X^{n}(s)-X^{n-1}(s)\right\|_{\mathbb{H}}^{2} d s,
\end{aligned}
$$

and this implies

$$
\mathbb{E}\left\|X^{n+1}(t)-X^{n}(t)\right\|_{\mathbb{H}}^{2} \leq\left[2 M^{2} t e^{2 \rho t} C_{f}+2 M^{2} t e^{2 \rho t} C_{g}\right] \int_{0}^{t} \sup _{0 \leq \tau \leq s} \mathbb{E}\left\|X^{n}(\tau)-X^{n-1}(\tau)\right\|_{\mathbb{H}}^{2} d s .
$$

Defining

$$
\Phi^{n}(t)=\sup _{0 \leq \theta \leq t} \mathbb{E}\left\|X^{n+1}(\theta)-Y^{n+1}(\theta)\right\|_{\mathbb{H}}^{2}
$$

we have

$$
\Phi^{n}(t) \leq C_{1} \int_{0}^{t} \Phi^{n-1}(s) d s \text { for } n \geq 2,
$$

for $C_{1}=2 T M^{2} e^{2 \rho T}\left[C_{f}+C_{g}\right]$. Consequently, by iteration we obtain

$$
\Phi^{n}(t) \leq \frac{C^{n-1} T^{n-1}}{(n-1) !} \Phi^{1}(t) \text { for all } n \geq 2 \text { and } t \in[0, T] .
$$

Since $X^{n+1}(t)=X^{n}(t), \forall t \in[-r, 0]$, the last estimate implies that $\left\{X^{n}\right\}_{n \in \mathbb{N}}$ is a solution of (3.1). But, this is straightforward taking into account that $X^{n}$ is defined by (3.3) and that $f$ and $g$ satisfies (H3)-(H8), so that, in particular, when $n \rightarrow \infty$,

$E\left\|\int_{0}^{t} R(t-s)\left(f\left(s, X_{s}^{n}-1\right)-f\left(s, X_{s}\right)\right) d s\right\|_{\mathbb{H}}^{2} \leq t M^{2} e^{2 \rho t} C_{f} \int_{0}^{t} E\left\|X^{n-1}(s)-X(s)\right\|_{\mathbb{H}}^{2} d s \rightarrow 0$,

and therefore $X$ is the unique (mild) solution of (3.1).

\section{Exponential decay of solutions in mean square}

As in this section we are interested in the exponential decay to zero in mean square of the mild solution to (3.1), we shall therefore assume that for each $T>0$ and for each $\varphi \in C\left(-r, 0 ; L^{2}(\Omega ; \mathbb{H})\right)$, problem $(3.1)$ possesses a unique mild solution according to Definition 3.1.

We first need to state the following conditions : 
Condition 1. The resolvent operator given by Theorem 2.2 satisfies

$$
\|R(t)\| \leq M e^{-\lambda t} \text { for } \forall t \geq 0, \quad \text { where } \quad M \geq 1, \lambda>0 .
$$

Condition 2. There exists a constant $D_{f} \geq 0$, such that, for any $x, y \in C\left(-r, T ; L^{2}(\Omega ; \mathbb{H})\right)$, and for all $t \geq 0$

$$
\int_{0}^{t} e^{m s}\left\|f\left(s, x_{s}\right)-f\left(s, y_{s}\right)\right\|_{\mathbb{H}}^{2} d s \leq D_{f} \int_{-r}^{t} e^{m s}\|x(s)-y(s)\|_{\mathbb{H}}^{2} d s, \quad \text { for all } \quad 0 \leq m \leq \lambda,
$$

and

$$
\int_{0}^{\infty} e^{\lambda s}\|f(s, 0)\|_{\mathbb{H}}^{2} d s<\infty
$$

Condition 3. There exists a constant $D_{g} \geq 0$, such that, for any $x, y \in C\left(-r, T ; L^{2}(\Omega ; \mathbb{H})\right)$, and for all $t \geq 0$

$$
\int_{0}^{t} e^{m s}\left\|g\left(s, x_{s}\right)-g\left(s, y_{s}\right)\right\|_{\mathcal{L}_{2}^{0}}^{2} d s \leq D_{g} \int_{-r}^{t} e^{m s}\|x(s)-y(s)\|_{\mathbb{H}}^{2} d s, \quad \text { for all } \quad 0 \leq m \leq \lambda,
$$

and

$$
\int_{0}^{\infty} e^{\lambda s}\|g(s, 0)\|_{\mathcal{L}_{2}^{0}}^{2} d s<\infty
$$

The following theorem shows the exponential dacay to zero in mean square, with an explicit decay rate $\gamma$.

Theorem 4.1. In addition to Conditions 1-3, assume that the mild solution $X(t)$ of system 3.1 corresponding to the initial function $\varphi \in C\left(-r, 0 ; L^{2}(\Omega ; \mathbb{H})\right)$, exists for all $t \geq-r$, and that $\lambda^{2}>6 M^{2}\left[D_{f}+D_{g}\right]$. Then, there exists a constant $\gamma>0$ such that

$$
\limsup _{t \rightarrow+\infty} \frac{1}{t} \log \mathbb{E}\|X(t)\|_{\mathbb{H}}^{2} \leq-\gamma
$$

In other words, every mild solution exponentially decays to zero in mean square.

Proof. Thanks to the fact that $\lambda^{2}>6 M^{2}\left[D_{f}+D_{g}\right]$, we can choose $\beta>0$ such that $\gamma=\lambda-\beta-6 M^{2}\left[D_{f}+D_{g}\right] \lambda^{-1}>0$. Then,

$$
\begin{aligned}
\mathbb{E}\|X(t)\|_{\mathbb{H}}^{2} \leq & 3 \mathbb{E}\|R(t) \varphi(0)\|_{\mathbb{H}}^{2}+3 \mathbb{E}\left\|\int_{0}^{t} R(t-s) f\left(s, X_{s}\right) d s\right\|_{\mathbb{H}}^{2} \\
& +3 \mathbb{E}\left\|\int_{0}^{t} R(t-s) g\left(s, X_{s}\right) d w(s)\right\|_{\mathbb{H}}^{2} .
\end{aligned}
$$

Therefore, by Condition 1 ,

$$
\begin{aligned}
\mathbb{E}\|X(t)\|_{\mathbb{H}}^{2} \leq & 3 M^{2} e^{-2 \lambda t} \mathbb{E}\|\varphi(0)\|_{\mathbb{H}}^{2}+3 M^{2} \int_{0}^{t} e^{-\lambda(t-s)} d s \int_{0}^{t} e^{-\lambda(t-s)} \mathbb{E}\left\|f\left(s, X_{s}\right)\right\|_{\mathbb{H}}^{2} d s \\
& +3 M^{2} \int_{0}^{t} e^{-\lambda(t-s)} d s \int_{0}^{t} e^{-2 \lambda(t-s)}\left\|g\left(s, X_{s}\right)\right\|_{\mathcal{L}_{2}^{0}}^{2} d s \\
\leq & 3 M^{2} e^{-2 \lambda t} \mathbb{E}\|\varphi(0)\|_{\mathbb{H}}^{2}+3 \lambda^{-1} M^{2} \int_{0}^{t} e^{-\lambda(t-s)} \mathbb{E}\left\|f\left(s, X_{s}\right)\right\|_{\mathbb{H}}^{2} d s \\
& +3 M^{2} \lambda^{-1} \int_{0}^{t} e^{-\lambda(t-s)}\left\|g\left(s, X_{s}\right)\right\|_{\mathcal{L}_{2}^{0}}^{2} d s
\end{aligned}
$$


and consequently,

$$
\begin{aligned}
e^{\lambda t} \mathbb{E}\|X(t)\|_{\mathbb{H}}^{2} \leq & 3 M^{2} \mathbb{E}\|\varphi(0)\|_{\mathbb{H}}^{2}+3 \lambda^{-1} M^{2} \int_{0}^{t} e^{\lambda s} \mathbb{E}\left\|f\left(s, X_{s}\right)\right\|_{\mathbb{H}}^{2} d s \\
& +3 M^{2} \lambda^{-1} \int_{0}^{t} e^{\lambda s}\left\|g\left(s, X_{s}\right)\right\|_{\mathcal{L}_{2}^{0}}^{2} d s
\end{aligned}
$$

and, for the chosen parameter $\beta$,

$$
\begin{aligned}
e^{(\lambda-\beta) t} \mathbb{E}\|X(t)\|_{\mathbb{H}}^{2} \leq & 3 M^{2} e^{-\beta t} \mathbb{E}\|\varphi(0)\|_{\mathbb{H}}^{2}+3 \lambda^{-1} M^{2} e^{-\beta t} \int_{0}^{t} e^{\lambda s} \mathbb{E}\left\|f\left(s, X_{s}\right)\right\|_{\mathbb{H}}^{2} d s \\
& +3 M^{2} e^{-\beta t} \int_{0}^{t} e^{\lambda s}\left\|g\left(s, X_{s}\right)\right\|_{\mathcal{L}_{2}^{0}}^{2} d s \\
\leq & 3 M^{2} e^{-\beta t} \mathbb{E}\|\varphi(0)\|_{\mathbb{H}}^{2}+3 \lambda^{-1} M^{2} \int_{0}^{t} e^{(\lambda-\beta) s} \mathbb{E}\left\|f\left(s, X_{s}\right)\right\|_{\mathbb{H}}^{2} d s \\
& +3 \lambda^{-1} M^{2} \int_{0}^{t} e^{(\lambda-\beta) s}\left\|g\left(s, X_{s}\right)\right\|_{\mathcal{L}_{2}^{0}}^{2} d s .
\end{aligned}
$$

Estimating the second and third terms in (4.2) in view of Condition 2 and Condition 3, there exists positive constant $C_{2}, C_{3}$ such that

$$
\begin{aligned}
\int_{0}^{t} e^{(\lambda-\beta) s} \mathbb{E}\left\|f\left(s, X_{s}\right)\right\|_{\mathbb{H}}^{2} d s \leq & 2 \int_{0}^{t} e^{(\lambda-\beta) s} \mathbb{E}\left\|f\left(s, X_{s}\right)-f(s, 0)\right\|_{\mathbb{H}}^{2}+2 \int_{0}^{t} e^{(\lambda-\beta) s} \mathbb{E}\|f(s, 0)\|_{\mathbb{H}}^{2} d s \\
\leq & 2 D_{f} \int_{-r}^{0} e^{(\lambda-\beta) s} \mathbb{E}\|\varphi(s)\|_{\mathbb{H}}^{2} d s+2 D_{f} \int_{0}^{t} e^{(\lambda-\beta) s} \mathbb{E}\|X(s)\|_{\mathbb{H}}^{2} d s \\
& +2 \int_{0}^{t} e^{(\lambda-\beta) s} \mathbb{E}\|f(s, 0)\|_{\mathbb{H}}^{2} d s \\
\leq & C_{2}+2 D_{f} \int_{-r}^{0} \mathbb{E}\|\varphi(s)\|_{\mathbb{H}}^{2} d s+2 D_{f} \int_{0}^{t} e^{(\lambda-\beta) s} \mathbb{E}\|X(s)\|_{\mathbb{H}}^{2} d s,
\end{aligned}
$$

and ,

$$
\int_{0}^{t} e^{(\lambda-\beta) s}\left\|g\left(s, X_{s}\right)\right\|_{L_{Q}^{0}}^{2} d s \leq C_{3}+2 D_{g} \int_{-r}^{0} \mathbb{E}\|\varphi(s)\|_{\mathbb{H}}^{2} d s+2 D_{g} \int_{0}^{t} e^{(\lambda-\beta) s} \mathbb{E}\|X(s)\|_{\mathbb{H}}^{2} d s .
$$

Thus, we have

$$
\begin{aligned}
e^{(\lambda-\beta) t} \mathbb{E}\|X(t)\|_{\mathbb{H}}^{2} \leq & 3 M^{2} \mathbb{E}\|\varphi(0)\|_{\mathbb{H}}^{2}+3 \lambda^{-1} M^{2}\left(C_{2}+C_{3}\right)+6 M^{2} \lambda^{-1}\left(D_{f}+D_{g}\right) \int_{-r}^{0} \mathbb{E}\|\varphi(s)\|_{\mathbb{H}}^{2} d s \\
& +6 M^{2} \lambda^{-1}\left(D_{f}+D_{g}\right) \int_{0}^{t} e^{(\lambda-\beta) s} \mathbb{E}\|X(s)\|_{\mathbb{H}}^{2} d s \\
= & C_{4}+6 M^{2} \lambda^{-1}\left(D_{f}+D_{g}\right) \int_{0}^{t} e^{(\lambda-\beta) s} \mathbb{E}\|X(s)\|_{\mathbb{H}}^{2} d s
\end{aligned}
$$

where $C_{4}$ is a suitable positive constant. The Gronwall Lemma conduces us to

$$
e^{(\lambda-\beta) t} \mathbb{E}\|X(t)\|_{\mathbb{H}}^{2} \leq C_{4} e^{6\left(D_{f}+D_{g}\right) M^{2} \lambda^{-1} t},
$$


and, consequently

$$
\begin{aligned}
\mathbb{E}\|X(t)\|_{\mathbb{H}}^{2} & \leq C_{4} e^{\left(6 M^{2}\left(D_{f}+D_{g}\right) \lambda^{-1}-\lambda+\beta\right) t} \\
& =C_{2} e^{-\gamma t}
\end{aligned}
$$

\section{Application}

In this section, we illustrate the abstract results proved in the previous sections by analyzing an example. Consider the following stochastic partial functional integrodifferential equation with finite delays:

$$
\left\{\begin{array}{l}
\frac{\partial}{\partial t} x(t, \xi)=\frac{\partial^{2}}{\partial \xi^{2}} x(t, \xi)+\int_{0}^{t} b(t-s) \frac{\partial^{2}}{\partial \xi^{2}} x(s, \xi)+\int_{-r}^{0} F(t, \theta, x(t+\theta, \xi)) d \theta \\
+G(t, x(t-r, \xi)) \dot{w}(t), \text { for } t \geq 0 \text { and } \xi \in[0, \pi], \\
x(t, 0)=0, \text { for } t \geq 0, \\
x(t, \pi)=0, \text { for } t \geq 0, \\
x(\theta, \xi)=x_{0}(\theta, \xi), \text { for } \theta \in[-r, 0] \text { and } \xi \in[0, \pi],
\end{array}\right.
$$

where $w(t)$ denotes a $\mathbb{R}$-valued Brownian motion, $F, G: \mathbb{R}^{+} \times \mathbb{R} \rightarrow \mathbb{R}$ are continuous functions, $b: \mathbb{R}^{+} \rightarrow \mathbb{R}$ is continuous and $x_{0}:[-r, 0] \times[0, \pi] \rightarrow \mathbb{R}$ is a given continuous function such that

$x_{0}(\cdot) \in L^{2}([0, \pi])$ is $\mathcal{F}_{0}$-measurable and satisfies $E\left\|x_{0}\right\|^{2}<\infty$.

Let $\mathbb{H}=L^{2}([0, \pi])$ with the norm $\|\cdot\|$ and $e_{n}:=\sqrt{\frac{2}{\pi}} \sin (n x), \quad(n=1,2,3, \cdots)$ denote the completed orthonormal basis in $\mathbb{H}$. Let $w(t):=\sum_{n=1}^{\infty} \sqrt{\lambda_{n}} \beta_{n}(t) e_{n}\left(\lambda_{n}>0\right)$, where $\beta_{n}(t)$ are one dimensional standard Brownian motion mutually independent on a usual complete probability space $\left(\Omega, \mathcal{F},\left\{\mathcal{F}_{t}\right\}_{t \geq 0}, \mathbb{P}\right)$.

Define $A: D(A) \subset \mathbb{H} \rightarrow \mathbb{H}$ by $A=\frac{\partial^{2}}{\partial z^{2}}$, with domain $D(A)=H^{2}([0, \pi]) \cap H_{0}^{1}([0, \pi])$. Then $A h=-\sum_{n=1}^{\infty} n^{2}<h, e_{n}>e_{n}, \quad h \in D(A)$, where $e_{n}, n=1,2,3, \cdots$, is the orthonormal set of eigenvectors of $A$. It is well-known that $A$ is the infinitesimal generator of a strongly continuous semigroup on $\mathbb{H}$, thus $(\mathbf{H 1})$ is true.

Let $B: D(A) \subset \mathbb{H} \rightarrow \mathbb{H}$ be the operator defined by

$$
B(t)(z)=b(t) A z \text { for } t \geq 0 \text { and } z \in D(A) \text {. }
$$

We assume that

(i) $F:[0,+\infty[\times[-r, 0] \times \mathbb{H} \rightarrow \mathbb{H}$ is a measurable function such that $\|F(t, \theta, x)-F(t, \theta, y)\|_{\mathbb{H}} \leq b_{1}\|x-y\|_{\mathbb{H}}, \quad \forall x, y \in \mathbb{H}, \quad t \geq 0, \quad \theta \in[-r, 0]$, where $b_{1}$ is 
a non-negative constant, and

$$
\int_{0}^{\infty} e^{\lambda s}\left(\int_{-r}^{0}\|F(s, \sigma, 0)\|_{\mathbb{H}}^{2} d \sigma\right) d s<\infty .
$$

(ii) $G::\left[0, \infty\left[\times C(-r, 0 ; \mathbb{H}) \rightarrow \mathcal{L}_{2}^{0}(\mathbb{K}, \mathbb{H})\right.\right.$ is a measurable function such that

$\|G(t, x)-G(t, y)\|_{\mathbb{H}} \leq b_{2}\|x-y\|_{\mathbb{H}}, \quad \forall x, y \in \mathbb{H}, \quad t \geq 0$, where $b_{2}$ is a non-negative constant, and

$$
\left.\int_{0}^{\infty} e^{\lambda s} \| G(s, 0)\right) \|_{\mathcal{L}_{2}^{0}}^{2} d s<\infty
$$

Let $C=C([-r, 0] ; \mathbb{H})$. Define the operators $f: \mathbb{R}^{+} \times \times[-r, 0] \times C \rightarrow \mathbb{H}$ and $g: \mathbb{R}^{+} \times C \rightarrow \mathbb{H}$ by

$$
\begin{gathered}
f(t, \phi)(\xi)=\int_{-r}^{0} F(t, \theta, \phi(\theta)(\xi)) d \theta \text { for } \xi \in[0, \pi] \text { and } \phi \in C, \\
g(t, \phi)(\xi)=G(t, \phi(-r)(\xi)) \text { for } \xi \in[0, \pi] \text { and } \phi \in C .
\end{gathered}
$$

If we put

$\left\{\begin{array}{c}u(t)=x(t, \xi) \text { for } t \geq 0 \text { and } \xi \in[0, \pi] \\ \varphi(\theta)(\xi)=x_{0}(\theta, \xi) \text { for } \theta \in[-r, 0] \text { and } \xi \in[0, \pi] .\end{array}\right.$

Then Eq. (5.1) takes the following abstract form

$$
\left\{\begin{array}{c}
d u(t)=A u(t) d t+\left[\int_{0}^{t} B(t-s) u(s) d s+f\left(t, u_{t}\right)\right] d t \\
+g\left(t, u_{t}\right) d w(t) \quad \text { for } t \in[0, T], \\
u_{0}(\cdot)=\varphi \in C=C([-r, 0] ; \mathbb{H}), r>0 .
\end{array}\right.
$$

Moreover, if $b$ is bounded and $C^{1}$ function such that $b^{\prime}$ is bounded and uniformly continuous, then (H1) and (H2) are satisfied and hence, by Theorem 2.2, Eq. (5.1) has a resolvent operator $(R(t))_{t \geq 0}$ on $\mathbb{H}$. Then it is straightforward to check that there exists a unique mild solution to (5.1).

If we assume, in addition, that

$$
\lambda^{2}>6 M^{2}\left[r b_{1}^{2}\left(e^{\lambda r}-1\right)+r b_{2}^{2}\left(e^{\lambda r}\right)\right]
$$

then, any mild solution to (5.1) decays exponentially to zero in mean square.

Acknowledgements. We would like to thank the referee for the interesting suggestion which improved the presentation of this paper.

This research has been partially supported by FEDER and Ministerio de Economía y Competitividad (Spain) under grant MTM2011-22411, and Consejerí de Innovación, Ciencia y Empresa (Junta de Andalucía) under Proyecto de Excelencia P12-FQM-1492. 


\section{References}

[1] A.Friedman. Stochastic Differential Equations and Applications. New York, Academic Press, 1975.

[2] M.A. Berger and V.J. Mizel. Volterra equations with ito integrals i. Journal of Integral Equations, 2:187-245, 1980.

[3] F. Brauer and P.V. Driessche. Models of transmission of disease with immigration of infectives. Math. BioSciences, 171:143-154, 2001.

[4] T.A. Bronikovski, J.E. Hall, and J.A. Nohel. Quantitative estimates for a nonlinear system of integrodifferential equations arising in reactor dynamics. SIAM J. Math. Anal., 3:567-588, 1972.

[5] T. Caraballo, M.J. Garrido-Atienza, and T. Taniguchi. The existence and exponential behavior of solutions to stochastic delay evolution equations with a fractional brownian motion. Nonlinear Analysis, 74(11):3671-3684, 2011.

[6] W.A. Coppel. Stability and Asymptotic Behavior of Differential Equations. Heath, Boston, 1965.

[7] W. Desch, R. Grimmer, and W. Schappacher. Some consideration for linear integrodifferential equations. Journal of Mathematical Analysis and Applications, 104:219-234, 1984.

[8] J. Golec and S. Sathananthan. Strong approximations of stochastic integrodifferential equations. Dynamics of Continuous, Discrete and Impulsive Systems, Series B, 8:139-151, 2001.

[9] R. Grimmer. Resolvent operators for integral equations in a banach space. Transactions of the American Mathematical Society, 273(1):333-349, 1984.

[10] R. Grimmer and A. J. Pritchard. Analytic resolvent operators for integral equations. Journal of Differential Equations, 50:234-259, 1983.

[11] K. Ito. On the existence and uniqueness of solutions of stochastic integral equations of the volterra type. Kodai Math. Journal, 2:158-170, 1979.

[12] J.Liang, J.H.Liu, and T.J.Xiao. Nonlocal problems for integrodifferential equations. Dynamics of Continuous, Discrete and Impulsive Systems, Series A, mathematical analysis, 15:815-824, 2008.

[13] D. Kannan. Random Integrodifferential Equations. Probability Methods and Related Topics, Volume 1, Academic Press, 1983.

[14] G.S. Ladde. Random Differential Inequalities. Academic Press, 1980.

[15] G.S. Ladde, V. Laksmikantham, and A.S. Vatsala. Monotone Iterative Techniques for Nonlinear Differential Equations. Pitman Advanced Publishing, Program, 1985. 
[16] V. Laksmikantham and M.R.M. Rao. Theory of Integro-Differential Equations. Gordon and Breach Publishers, 1995.

[17] L.Arnold. Stochastic Diferential Equations. John Wiley, 1974.

[18] J.J. Levin and J.A. Nohel. The integro-differential equations of a class of nuclear reactors with delayed neutrons. Arch. Rat. Mech. Anal., 31:151-172, 1968.

[19] R.K. Miller. An integro-differential equation for heat conductors with memory. $J$. Math. Anal. Applications, 66:313-333, 1978.

[20] B. Oksendal. Stochastic Differential Equations. Springer, 1998.

[21] J. Pruss. Evolutionary Integral Equations and Applications. Birkhauser, 1993. 